ESAIM: PROCEEDINGS AND SURVEYS, November 2014, Vol. 46, p. 213-216

ECIT 2012 - Witold Jarczyk, Daniele Fournier-Prunaret, João Manuel Gonçalves Cabral

\title{
PIECEWISE EXPANDING MAPS: COMBINATORICS, DYNAMICS AND REPRESENTATION OF RATIONAL NUMBERS
}

\author{
Cristina Serpa And Jorge Buescu ${ }^{1}$
}

\begin{abstract}
We establish combinatorial properties of the dynamics of piecewise increasing, continuous, expanding maps of the interval such as description of periodic and pre-periodic points, primitiveness of truncated itineraries and length of pre-periodic itineraries. We include a relation between the dynamics of a family of circle maps and the properties of combinatorial objects as necklaces and words. We identify in a natural way each periodic orbit with an aperiodic necklace. We show the relevance of this combinatorial approach for the representation of rational numbers and for the orbit structure of pre-periodic points.

Keywords: Piecewise expanding map, periodic point, pre-periodic point, representation of rational numbers

Résumé. Nous établissons des propriétés combinatoires de la dynamique des fonctions de l'intervalle croissant, continu et expansif par morceaux tels que description des points périodiques et pré-périodiques, primitif itinéraires de troncs et de la durée d'itinéraires pré-périodiques. Nous inclure une relation entre la dynamique d'une famille de fonctions du cercle et les propriétés des objets combinatoires que des colliers et des mots. Nous identifions de façon naturelle chaque orbite périodique avec un apériodique collier. Nous montrons la pertinence de cette approche combinatoire pour la représentation des nombres rationnels et de la structure de l'orbite des points de pré-périodiques.

Mots-clés: Fonction expansif par morceaux, point périodique, point pré-périodique, représentation des nombres rationnels
\end{abstract}

\section{INTRODUCTION}

Chaotic maps have three main properties: unpredictability, indecomposability and an element of regularity (see e.g. [3]). The first comes from sensitive dependence on initial conditions, the second is due to the impossibility of decomposing into two or more subsystems and the last one arises from the density of periodic points. However, unpredictability is compatible with the deterministic nature of chaotic systems. In fact, it is possible to study the long time behavior of this kind of systems using tools from ergodic theory (see e.g. [1]). Using combinatorics and symbolic dynamics, we devote particular attention to a family of chaotic maps for which it is possible to obtain partial predictability for long-term behavior.

Fotiades and Boudourides (see [4]) extended the work of Milnor and Thurston [7] on symbolic dynamics of piecewise monotone maps to a family $\mathcal{M}$ of piecewise monotone, not necessarily continuous, expansive interval maps.

Mathematics Subject Classification 2010: 37E15, 37E05, 37E10.

1 Cristina Serpa Centro de Matemática e Aplicações Fundamentais, Departamento de Matemática, Faculdade de Ciências, Universidade de Lisboa, 1749-016 Lisboa, Portugal, Tel: 00351 2175100293, E-mail: mcserpa@fc.ul.pt,

Jorge Buescu Centro de Matemática e Aplicações Fundamentais, Departamento de Matemática, Faculdade de Ciências, Universidade de Lisboa, 1749-016 Lisboa, Portugal, Tel: 00351 2175100293, E-mail: jsbuescu@fc.ul.pt

(C) EDP Sciences, SMAI 2014 
Definition 0.1. $f:[0,1] \rightarrow[0,1]$ belongs to $\mathcal{M}$ if there exists a partition $0=a_{0}<a_{1}<\cdots<a_{r}=1$ $(r \geq 2)$ of $[0,1]$ such that $f \mid\left(a_{i-1}, a_{i}\right)(i=1,2, \ldots, r)$ is a monotone $C^{1}$ function satisfying the following Markov condition: for every $i=1,2, \ldots, r$, there exist $p(i), q(i) \in\{0,1, \ldots, r\}$ with $p(i)<q(i)$ such that $f\left(a_{i-1}, a_{i}\right)=\left(a_{p(i)}, a_{q(i)}\right)$ and there is $\lambda>1$ such that $\left|f^{\prime}(x)\right| \geq \lambda$ for almost every $x \in[0,1]$.

We remark that the results of Fotiades and Boudourides for topological conjugacies of maps remain valid under slightly weaker conditions. We consider the analog of their results within a broader class of functions $\mathcal{M}$ which are not necessarily piecewise $C^{1}$ but only piecewise continuous. The expansivity condition is also weakened.

Definition 0.2. A continuous map $f: X \rightarrow X$ on a metric space $(X, d)$ is called expanding if there exist constants $\epsilon>0$ and $\lambda>1$ such that for all $x, y \in X$

$$
d(x, y)<\epsilon \Rightarrow d(f(x), f(y)) \geq \lambda d(x, y) .
$$

$\lambda$ is called the expansion factor of $f$.

Theorem 0.3. (Fotiades and Boudourides) Let $f \in \mathcal{M}$ with partition $0=a_{0}<a_{1}<\cdots<a_{r}=1$. Let $T \in \mathcal{M}$ be the map with partition $0=1 / r<2 / r<\cdots<r-1 / r<1$ which is linear in each interval $[(i-1) / r, i / r]$ and $T((i-1) / r, i / r)=(p(i) / r, q(i) / r)$. Furthermore, $T \mid[(i-1) / r, i / r]$ is of the same monotonicity type as $f \mid\left(a_{i-1}, a_{i}\right)$ and it is continuous, from the right or from the left at $i / r$, when $f$ is continuous, from the right or from the left at $a_{i}$, respectively. Then $f$ and $T$ are topologically conjugate, i.e., there exists a homeomorphism $h:[0,1] \rightarrow[0,1]$ such that $h \circ f=T \circ h$.

This result states that the diagram

$$
\begin{array}{cccc}
{[0,1]} & \stackrel{f}{\rightarrow} & {[0,1]} \\
h \downarrow & & \downarrow h \\
{[0,1]} & \rightarrow & {[0,1]}
\end{array}
$$

is commutative.

By this result the topological properties of maps belonging to $\mathcal{M}$ can be derived from the study of the piecewise linear case. Topological conjugacy ensures that properties related to periodic and pre-periodic are preserved. The next result is the specification of Theorem 0.3 for the piecewise increasing case.

Corollary 0.4. Let $f \in \mathcal{M}$ with partition $0=a_{0}<a_{1}<\cdots<a_{r}=1$ of $[0,1]$ such that $\left.f\right|_{\left[a_{i-1}, a_{i}\right]}$ $(i=1,2, \ldots, r)$ is an increasing, continuous and expanding function satisfying $f\left(a_{i-1}, a_{i}\right)=(0,1)$, for every $i=1,2, \ldots, r$. Then $f$ is topologically conjugate to the map

$$
g_{a}(x)= \begin{cases}a x(\bmod 1) & , \text { if } x \in[0,1) \\ 1 & , \text { if } x=1,\end{cases}
$$

where $a=r+1$.

By Corollary 0.4 there exists a homeomorphism $h$ such that

$$
h \circ g=f \circ h .
$$

Denoting by $I_{j}=[j / a,(j+1) / a)$, the interval map $g_{a}$ may be written as $g_{a}(x)=a x-j$ if $x \in I_{j}$, where $j$ ranges from 0 to $a-1$. In terms of symbolic dynamics, following the work of Milnor and Thurston [7], we associate the symbol $j$ to an $x \in I_{j}$.

We now introduce some concepts of formal languages defined in [2], and [8]

Definition 0.5. An alphabet $A$ is a finite nonempty totally ordered set of symbols (letters). A word or string over an alphabet $A$ is a finite sequence of symbols taken from $A$. We denote by $\sum$ the set of words over an alphabet. A necklace is the equivalence class of a word under a circular shift. 
Definition 0.6. A word $u \in \sum$ is primitive, or aperiodic, if

$$
\left.u=z^{n} \Rightarrow n=1 \text { (and hence } u=z\right)
$$

where $z \in \sum$ and $z^{n}$ means the $n$-fold catenation of the word $z$.

The next result is proved in [9]. We supply a sketch of the proof.

Proposition 0.7. Consider the map $g_{a}$. Its $m$-periodic points are of the form

$$
x=\frac{\sum_{k=1}^{m} j_{k} a^{m-k}}{a^{m}-1},
$$

where $a \geq 2$ is an integer and $0 \leq j_{k}<a$, and the pre-periodic points are of the form

$$
x=\frac{\sum_{k=1}^{m} j_{k} a^{m-k-n}}{a^{m}-1}+\sum_{k=1}^{n} i_{k} a^{-k},
$$

where $0 \leq j_{k}<a$ is an integer and $0 \leq i_{k}<a$.

To prove the statement it suffices to compute the first $n$ iterates of the dynamical system. After this preperiodic points are found through a path of pre-images of periodic points.

Remark 0.8. If an m-truncated itinerary of a periodic point with prime period $m$ is considered as a word of a formal language, then it is a primitive word in the sense of Definition 0.6.

Combinatorial properties of the map $g_{a}$ are a consequence of the existence of a bijection between the set of aperiodic necklaces (with primitive words) of lenght $m$ from an alphabet with a words and the set of periodic orbits of period $m$ of the map $g_{a}$.

$A$ consequence of (0.6) is the existence of a finite base-a representation for every rational number.

Theorem 0.9. Let $a \geq 2$ be a positive integer and $x \in \mathbb{Q}$. Then there exist integers $m \geq 1,0 \leq j_{1}, j_{2}, \ldots, j_{m}<$ $a, n \geq 1,-a<i_{1}, i_{2}, \ldots, i_{n}<a$ and $s$ such that

$$
x=\frac{\sum_{k=1}^{m} j_{k} a^{m-k-n}}{a^{m}-1}+\sum_{k=s}^{n} i_{k} a^{-k} .
$$

The proof of this theorem results from the fact that, by the properties of the map $g_{a}$, the set of rational numbers in the domain is the set of pre-periodic points of the this map. form

From this representation it is easily shown that each rational number may be represented as a fraction of the

$$
x=\frac{b}{a^{n}\left(a^{m}-1\right)},
$$

for every positive integer $a \geq 2$ and some $n, m \in \mathbb{N}$ and $b \in \mathbb{Z}$ which depend on $x$.

Corollary 0.10. Let $a \geq 2$ be a positive integer. Then every positive integer has multiples of the form $a^{n}\left(a^{m}-1\right)$, where $n \in \mathbb{N}_{0}$ and $m \in \mathbb{N}$. We say $a^{n}\left(a^{m}-1\right)$ is the a-base least multiple (a-blm) of a number if it is the least possible multiple of this form.

This construction bears a close relation to the theory of $p$-adic numbers [5], as already remarked by Hehner and Horspool [6] in an analogous construction. 
From the dynamical point of view the main advantage of writing a number in the finite representation $(0.7)$ is that it supplies complete information about its orbit under the map $g_{a}$. It is thus useful to represent rational numbers in this way.

We now provide an algorithm to convert the usual representation $p / q$ of rational numbers into the representation $(0.7)$. Without loss of generality we consider a number in the interval $[0,1)$.

Let $p / q \in \mathbb{Q} \cap[0,1)$ be an irreducible fraction.

Step 1: find the $a$-base least multiple of $q$.

Step 2: multiply the numerator and the denominator by the adequate number such that

$$
\frac{p}{q}=\frac{P}{a^{n}\left(a^{m}-1\right)}
$$

Step 3: perform successive divisions by $\left(a^{m}-1\right), a^{n}, a^{n-1}$, etc.

Bearing in mind the existence of the conjugacy (0.3) the next result is immediate.

Theorem 0.11. Let $f \in \mathcal{M}$ be in conditions of Corollary 0.4. The pre-periodic points of $f$ are of the form

$$
x=h\left(\frac{\sum_{k=1}^{m} j_{k} a^{m-k-n}}{a^{m}-1}+\sum_{k=s}^{n} i_{k} a^{-k}\right),
$$

It is now possible to construct complete symbolic itineraries for pre-periodic points, including the description of the eventual period. If the point is written in the form (0.10), then all its dynamical behavior is known. Now let $x \in[0,1]$ and suppose the homeomorphism $h$ is known. If $h^{-1}(x) \in[0,1] \cap \mathbb{Q}$ the point is pre-periodic and it is possible to estimate how many iterates are needed for the point to reach a periodic itinerary and know the period of the corresponding orbit. Let $p / q \in \mathbb{Q} \cap[0,1)$ be the irreducible fraction of $h^{-1}(x)$. Multiplying the numerator and the denominator $p / q$ by the smallest value such that the new equivalent fraction is of the form $b /\left(a^{n}\left(a^{m}-1\right)\right)$, we conclude that $n$ is at most the number of iterates of the pre-periodic part of the orbit and $m$ is the period of the periodic part of the itinerary.

Note that if $h^{-1}(x) \in[0,1] \backslash \mathbb{Q}$ then its orbit is dense in $[0,1]$. In this case, and with this method, no further orbit prediction is possible to describe. This is inherent to the unpredictability of chaotic systems.

\section{Acknowledgments:}

JB acknowledges partial support from FCT through Financiamento Base 2010-ISFL-1-209. CS acknowledges support from FCT through grant SFRH/77623/2011.

\section{REFERENCES}

[1] Abraham Boyarsky, Paweł Gra, Laws of Chaos, Invariant Measures and Dynamical Systems in One Dimension, Probability and Its Applications, Birkhuser, 1997.

[2] Jean Berstel, Dominique Perrin, The origins of combinatorics on words, Eur. J. Combin., 28 (2007), pp. $996-1022$.

[3] Robert L. Devaney, An Introduction to Chaotic Dynamical Systems, 2nd Ed., Addison-Wesley Publishing Company, 1989.

[4] Nikos A. Fotiades and Moses A. Boudourides, Topological conjugacies of Piecewise Monotone Interval Maps, Internat. J. Math. Math. Sci., Vol. 25, 2 (2001), pp 119-127.

[5] Fernando Q. Gouva, p-adic Numbers - An Introduction, 2nd Ed., Springer, 2000.

[6] E. C. R. Hehner and R. N. S. Horspool, A New Representation of the Rational Numbers for Fast Easy Arithmetic, SIAM J. Comput, Vol 8, 2 (1979), pp. 124-134.

[7] John Milnor and William Thurston, On iterated maps of the interval, Lect. Notes Math., Springer, Vol. 1342 (1988), pp. $465-563$.

[8] G. Rozenberg, A. Salomaa Eds, Handbook of Formal Languages, Word Language Grammar, Vol. 1, Springer, 1997.

[9] Cristina Serpa, Jorge Buescu, Combinatorics of piecewise expanding interval maps, submitted for publication. 